INPLASY

PROTOCOL

To cite: Luo et al. The effect of acupoint catgut embedding and drug therapy on hyperlipidemia: a metaanalysis of randomized controlled trials. Inplasy protocol 202220019. doi: 10.37766/inplasy2022.2.0019

Received: 08 February 2022

Published: 08 February 2022

Corresponding author: Jing Luo

luojingne@163.com

Author Affiliation:

None

Support: None.

Review Stage at time of this submission: Preliminary searches.

Conflicts of interest: None declared.

\section{The effect of acupoint catgut embedding and drug therapy on hyperlipidemia: a meta-analysis of randomized controlled trials}

Luo, J1; Zhang, C2; Wu, X3; Ren, R4.

Review question / Objective: Acupoint catgut embedding and drug treatment of hyperlipidemia compared, which is better. Condition being studied: Hyperlipidemia.

Information sources: Two authors (JL and CZ) will examine the publications independently and extract data according to predefined criteria. RCTs will be assessed for the methodology, study design, inclusion and exclusion criteria, and outcome measures. The methodological quality of each RCT will be recorded for method of randomization, blinding, protocol violation, and allocation concealment Any disagreement will be resolved by consensus discussions with the senior member of the review team (XP W). Data to collect includes intervention and control measures, measured outcomes and statistical significant difference with regards to chewing gum.

INPLASY registration number: This protocol was registered with the International Platform of Registered Systematic Review and Meta-Analysis Protocols (INPLASY) on 08 February 2022 and was last updated on 08 February 2022 (registration number INPLASY202220019).

\section{INTRODUCTION}

Review question / Objective: Acupoint catgut embedding and drug treatment of hyperlipidemia compared, which is better.

Condition being studied: Hyperlipidemia.

\section{METHODS}

Search strategy: \#1 "Hyperlipidemias" [MeSH]

\#2 "Hyperlipemia"[Title/Abstract] OR "Hyperlipemias"[Title/Abstract] OR "Hyperlipidemia" [Title/Abstract] OR "Lipidemia" [Title/Abstract] OR 
"Lipidemias" [Title/Abstract] OR "Lipemia" [Title/Abstract] OR "Lipemias" [Title/ Abstract]

\#3 \#1 OR \#2

\#4 "Acupuncture"[MeSH] OR "acupotomy" [Title/Abstract]OR "acupotomies"[Title/ Abstract] OR "acupoint" [Title/Abstract]OR "acupoint catgut embedding" [Title/ Abstract] OR "acupoint thread embedding" [Title/Abstract] OR "catgut implantation" [Title/Abstract] OR "acupoints thread embedding"[Title/Abstract]OR "catgu-t embedding" [ Title/Abstract] OR "embedding therapy"[Title/Abstract] OR "thread-embedding" [Title/Abstract] \#5\#3 AND \#4.

Participant or population: Pantients who with hyperlipidemia.

Intervention: Acupoint Catgut Embedding.

\section{Comparator: Drug therapy.}

Study designs to be included: RCT.

Eligibility criteria: Cohort studies and other studies were excluded.

Information sources: Two authors (JL and CZ) will examine the publications independently and extract data according to predefined criteria. RCTs will be assessed for the methodology, study design, inclusion and exclusion criteria, and outcome measures. The methodological quality of each RCT will be recorded for method of randomization, blinding, protocol violation, and allocation concealment Any disagreement will be resolved by consensus discussions with the senior member of the review team (XP W). Data to collect includes intervention and control measures, measured outcomes and statistical significant difference with regards to chewing gum.

Main outcome(s): The relationship between hyperlipidemia and acupoint catgut embedding.

Quality assessment / Risk of bias analysis: Any disagreement will be resolved by consensus discussions with the senior member of the review team (MJ X and XP W).

Strategy of data synthesis: Using the standard method recommended by the Cochrane Collaboration, the outcome index included in the meta-analysis was pain. The random-effect model was predetermined to be most suitable for this meta-analysis, considering both catgut embedding and analgesia.

Subgroup analysis: Comparison of different drug and point.

Sensitivity analysis: Conduct sensitivity analysis.

Language: English.

Country(ies) involved: China.

Keywords: acupoint catgut embedding, hyperlipidemia.

Contributions of each author:

Author 1 - Jing Luo.

Email: luojingne@163.com

Author 2 - Chen Zhang.

Email: chan299@163.com

Author 3 - Xiaoping Wu.

Email: 95255996@qq.com

Author 4 - Rongrong Ren.

Email: 103618894@qq.com 